\title{
Andiamo oltre?
}

Vittorio Coletti

PUBBLICATO: 25 GENNAIO 2019

\section{Quesito:}

Due domande riguardano la preposizione impropria oltre e la locuzione congiunzionale oltre che.

\section{Andiamo oltre?}

Per la preposizione il dubbio è sulla sua reggenza: vuole o no un'altra preposizione propria prima dell'elemento nominale che la segue, e se sì, quale?

Oltre ha, come avverbio e poi come preposizione, due valori, ereditati dal latino ultra da cui discende, di prosecuzione nello spazio e nel tempo: nel titolo italiano di un famoso romanzo di Harper Lee, Il buio oltre la siepe (da cui fu tratto un altrettanto famoso film), oltre ha valore spaziale; nel titolo italiano del celebre saggio di un noto medico americano, Raymond Moody, La vita oltre la vita, oltre ha valore temporale. Invece la locuzione preposizionale oltre a significa 'in aggiunta a', 'più di', come in: "oltre alla casa vende anche il garage"; ma se a oltre segue un numerale non ci vuole la preposizione di raccordo: "non lo sento da oltre un anno", "guadagna oltre duemila euro al mese".

Ma se dopo oltre c'è un pronome personale? La regola delle preposizioni improprie che richiedono, in tal caso, un collegamento tramite preposizione propria è rispettata: occorre di per i due significati spaziali o temporali ("la donna forzò un sorriso e passò oltre di noi sulla scala", E. De Luca, I pesci non chiudono gli occhi; "un pegno che non durerà oltre di noi", Milo de Angelis, Ė entrato qui) e a per quello di 'in aggiunta' ("Al Ministero, oltre a lui, c'erano non so quanti suoi fratelli, cugini, parenti, compari e compaesani", C. Levi, L'Orologio).

Non è sempre stato così, per la verità, e in passato di si poteva trovare col valore di aggiunta ("Sua Eccellenza si parti di Livorno accompagnato, oltre di noi, da tutti quei della nazione", si legge in un Ragguaglio del i687 scovato su Google Libri) e a con quello temporale: Boccaccio, Teseida, libro x: "E se pur fia la mia disavventura/ di vivere oltre a te". Oggi la lingua ha semplificato un po' le cose e il GRADIT segnala che se oltre a è comune, oltre di, nello stesso significato, è ormai di basso uso.

Dunque davanti a nome la sola preposizione oltre introduce i significati di 'prosecuzione nello spazio o nel tempo' e la locuzione preposizionale oltre a quello di 'in aggiunta a'.

Per questo, venendo a una delle due domande, che chiede se è più giusto "concordare lezioni oltre all'orario previsto" o "oltre l'orario previsto", si deve rispondere che le due forme introducono due significati diversi: "oltre all'orario", significa lezioni in più rispetto all'orario previsto; "oltre l'orario" vuol dire dopo, alla fine dell'orario previsto.

Si pensi anche, per chiarezza e sintesi del comportamento attuale della lingua, a locuzioni fisse come oltre a questo, a ció, munite di preposizione propria, il cui significato è 'in aggiunta', e a parole composte come oltreconfine o oltre vita, senza preposizione, in cui il significato di oltre è spaziale o temporale.

Fa però eccezione la locuzione oltre tutto (oggi anche univerbata in oltretutto) 'oltre al resto', che è ben più frequente (tranne che in alcune particolari varietà regionali, come quella romana), della, per altro più antica,oltre a tutto,che rispettava la reggenza standard per il significato di oltre come aggiunta.

Quanto alla domanda sulla locuzione congiunzionale: oltre che, introduttiva di una "proposizione aggiuntiva di forma implicita" (Serianni 1989), e oltre a sono perfettamente sinonimi, per cui si può dire tanto "oltre che dare fastidio..." quanto "oltre a dare fastidio...". A volte il verbo all'infinito è sottinteso e in tal caso è d'obbligo che: "oltre che (essere) bello è anche buono". Nell'Isola di Arturo della Morante la frase "Oltre che di adulazioni, io vivevo del tutto digiuno di baci e di carezze", se svolta interamente, sarebbe cosi: "oltre che (vivere del tutto digiuno) di adulazioni, io vivevo del tutto digiuno di baci e di carezze". In certi casi l'ellissi è tale che la locuzione si sgancia quasi del tutto dai dintorni sintattici e si avvicina al valore testuale di "e inoltre", come in questo passo 
dalla Pelle di Malaparte: "Si illudevano di aver trovato... una complicità segreta, un nuovo patto di natura morale e sociale oltreche sessuale".

\section{Cita come:}

Vittorio Coletti, Andiamo oltre?, "Italiano digitale", 2019, VIII, 2019/1 (gennaio-marzo) , pp. 11-12. DOI: $10.35948 / 2532-9006 / 2019.3062$

Copyright 2019 Accademia della Crusca

Pubblicato con licenza creative commons CC BY-NC-ND 